\title{
Destabilizing excitonic insulator phase by pressure tuning of exciton-phonon coupling
}

\author{
Sukanya Pal $\odot,{ }^{1, *}$ Shivani Grover $\odot,{ }^{2,3,},{ }^{*}$ Luminita Harnagea $\odot,{ }^{4}$ Prachi Telang, ${ }^{4}$ Anupam Singh,,${ }^{4}$ D. V. S. Muthu, ${ }^{1}$ \\ U. V. Waghmare $\odot,{ }^{3}$ and A. K. Sood $\odot^{1, \dagger}$ \\ ${ }^{1}$ Department of Physics, Indian Institute of Science, Bangalore 560012, India \\ ${ }^{2}$ Chemistry and Physics of Materials Unit, Jawaharlal Nehru Centre for Advanced Scientific Research, Bangalore 560064, India \\ ${ }^{3}$ Theoretical Sciences Unit, Jawaharlal Nehru Centre for Advanced Scientific Research, Bangalore 560064, India \\ ${ }^{4}$ Department of Physics, Indian Institute of Science Education and Research, Pune, Maharashtra 411008, India
}

(Received 25 March 2020; revised 11 September 2020; accepted 12 October 2020; published 4 November 2020)

\begin{abstract}
$\mathrm{Ta}_{2} \mathrm{NiSe}_{5}$ is an excitonic insulator (EI) exhibiting bound electron-hole pairs condensed at room temperature, which transforms to a small-gap semiconducting state above $T=325 \mathrm{~K}$ simultaneously undergoing a phononrelated structural transition. Despite the clear experimental evidence for strong exciton-phonon coupling, its role and the origin of EI state in terms of BCS versus Bose-Einstein condensation mechanisms are unclear. Motivated by the tunability of these mechanisms with pressure, we report Raman experiments under pressure of $\mathrm{Ta}_{2} \mathrm{NiSe}_{5}$ and first-principles theoretical analysis of two pressure-induced transitions at 1 and $3 \mathrm{GPa}$. We present a simple method to derive the exciton-phonon coupling within density functional theory and show using a model Hamiltonian that reducing strength of this coupling relative to electronic gap and phonon frequency destabilizes the EI state with pressure. In addition to connecting with the Raman anomalies observed under pressure, our simple picture explains the recently observed phonon-coupled state of exciton condensate.
\end{abstract}

DOI: 10.1103/PhysRevResearch.2.043182

\section{INTRODUCTION}

An excitonic insulating state arises when electrons and holes in a small band overlap semimetal or a small band-gap semiconductor forms bound pairs (excitons) due to attractive Coulomb interaction and poor screening, condensing into a quantum state with macroscopic phase coherence. Excitonic condensation in semiconducting systems involves Bose-Einstein condensation (BEC) of preformed excitons, and that in semimetallic systems involves a mechanism analogous to the BCS pairing of electrons in superconductors [1-4]. While excitonic insulating states were conceptualized half a century ago [1], they have attracted a great deal of attention recently with growing evidence for their experimental realization. A transition-metal dichalcogenide $\mathrm{Ta}_{2} \mathrm{NiSe}_{5}$ [5] has been proposed recently as an EI at room temperature and pressure by Lu et al. [6], which does not exhibit any charge density wave transition, making it an ideal material system for investigation of the EI phase.

$\mathrm{Ta}_{2} \mathrm{NiSe}_{5}$ (TNSe) is a direct band-gap $\left(E_{g} \leqslant 50 \mathrm{meV}\right)$ semiconductor at high temperature as reflected in optical, transport, and specific heat measurements [6]. It undergoes a second-order phase transition upon cooling to a mono-

\footnotetext{
*These authors contributed equally to this work.

†asood@iisc.ac.in
}

Published by the American Physical Society under the terms of the Creative Commons Attribution 4.0 International license. Further distribution of this work must maintain attribution to the author(s) and the published article's title, journal citation, and DOI. clinic structure with an anomaly in electrical resistivity at $T_{c} \approx 325 \mathrm{~K}$ [7]. Below $T_{c}$, it is shown to exhibit weakly dispersed, flat valence and conduction bands, a signature of the EI state [8]. The ground state is characterized by the excitonic coupling between the $\mathrm{Ni}(3 d)-\mathrm{Se}(4 p)$ hole states in the valence band and the $\mathrm{Ta}(5 d)$ electronic states in the conduction band at $\Gamma$ point [7-11]. The excitonic condensation drives a second-order structural transition from orthorhombic $(\mathrm{Cmcm})$ to monoclinic $(C 2 / c)$ structure through a slight distortion of Ta double chains with respect to single $\mathrm{Ni}$ chains while maintaining the unit cell size [12]. Electronic structure of TNSe calculated with simple density functional theory show that it is metallic supporting a BCS-like mechanism involving phonons. Theoretical analysis of the Falikov-Kimble model within density matrix renormalization group showed that the high-temperature state is gapped, with preformed excitons stabilized by a strong electron-hole coupling [13], supporting the Bose-Einstein condensation (BEC) scenario. However, the origin of this strong coupling or a possibility of BCS mechanism are presently unclear.

There have been several recent works demonstrating a clear evidence for strong coupling between phonons and excitons (or electrons). A spatially extended state of coupled exciton and phonons was associated with large oscillator strength attributed to the giant exciton Fano resonance observed in spectroscopic ellipsometry [14]. Using nonlinear excitations with laser pulses, Werdehausen et al. [11] established the presence of $A_{1 g}$ phonon-coupled state of the exciton condensate. A recent inelastic x-ray scattering (IXS) experiment revealed that strong electron-optical phonon coupling results in enhanced linewidth of the transverse optical mode above $T_{c}$ and softening of transverse acoustic mode below $T_{c}$ relevant to the concurrent structural and EI to semiconductor 
transition [15]. Infrared phonon spectroscopic measurements of Larkin et al. [16] suggest strongly overlapping excitonphonon complexes correlating with the excitonic insulating state and the structural phase transition. Results of a recent study [17] based on scanning tunneling spectroscopy and theoretical calculations indicate that excitonic condensation in TNSe may be close to the BCS regime. Electronic band gap and phonons can be tuned with pressure, and BCS versus BEC mechanisms of excitonic insulating state can thus be accessed systematically with pressure [6].

A recent $\mathrm{x}$-ray diffraction experiment shows that a transition from EI to semiconductor phase is observed at pressure $P_{c 1} \approx 1.5 \mathrm{GPa}$ at room temperature [18]. With further increase in pressure, another structural phase transition is observed at $P_{c 2} \approx 3.0 \mathrm{GPa}$ which takes the system from C-centered orthorhombic $(\mathrm{Cmcm})$ to primitive orthorhombic (Pmnm) structure and the system becomes semimetallic above $P_{c 2}$. This pressure-induced reversible transformation at $P_{c 2}$ is a first-order phase transition, caused by coherent sliding of the weakly coupled structural layers. With further increase in pressure and decrease in temperature, the system goes into a superconducting state around $P \approx 8 \mathrm{GPa}$ with the superconducting transition temperature close to $\approx 1.2 \mathrm{~K}$ [18]. Raman spectroscopy is an effective tool to study coupled structural and electronic transitions under pressure, as the changes in pressure coefficients of mode frequencies and Raman line widths reflect changes in the electron-phonon coupling (EPC) and disappearance and/or appearance of other modes reveal changes in the structural symmetry.

Here, we use a combination of Raman experiments, first-principles density functional theory (DFT), and model Hamiltonian analysis of exciton-phonon coupling to confirm the EI to semiconductor transition in $\mathrm{Ta}_{2} \mathrm{NiSe}_{5}$ as a function of pressure and uncover its mechanism. We present a simple scheme within DFT to estimate the exciton-phonon coupling and use it within a BCS-like model with TNSe-specific parameters as a function of pressure. A simple mean-field analysis (similar to that of Falikov-Kimble model [19]) of this model is used to predict the EI to normal semiconductor transition (at $P_{c 1}$ ), connecting with our observed Raman anomalies that constitute readily measurable signatures of the transition.

\section{EXPERIMENTAL DETAILS}

Single crystals of $\mathrm{Ta}_{2} \mathrm{NiSe}_{5}$ were grown by chemical vapor transport method using $\mathrm{I}_{2}$ as the transporting agent. The weighing, cutting, mixing, and grinding of the precursors were carried out in an Ar-filled glove box where the moisture and oxygen is maintained below $0.1 \mathrm{ppm}$. The growth experiments were carried out in two steps. In the first step, the precursor materials Ta powder (Sigma Aldrich, 99.9\%, metals basis), Ni wire (Sigma Aldrich, 99.9\%, metals basis), and Se shot (Alfa Aesar, 99.999+\%, metals basis), taken in appropriate amounts (Ta:Ni:Se $=2: 1: 5.05 ; 1 \% \mathrm{Se}$ in excess), were placed in an alumina crucible and subsequently sealed under vacuum in a quartz ampoule. This was slowly heated to $750{ }^{\circ} \mathrm{C}$, with a rate of $50{ }^{\circ} \mathrm{C} / \mathrm{h}$, kept there for $48 \mathrm{~h}$, and then cooled down to room temperature at a rate of $100{ }^{\circ} \mathrm{C} / \mathrm{h}$. The polycrystalline samples prepared in this way were finely ground in the glove box. The powder was then loaded and sealed under vacuum in a quartz ampoule together with the transporting agent (iodine, $5 \mathrm{mg} / \mathrm{cm}^{3}$ ). Subsequently, the ampoule has been placed in a furnace with a gradient of temperature between $870{ }^{\circ} \mathrm{C}$ (source zone T1) and $820{ }^{\circ} \mathrm{C}$ (sink zone T2). After 12 days, needle-shaped single crystals with typical dimensions of 1-15 $\mathrm{mm}$ were obtained. The crystal structure, morphology, and chemical composition of the single crystals were determined using $\mathrm{x}$-ray powder diffraction [Bruker D8 diffractometer $\left(\mathrm{Cu} \mathrm{K} \alpha_{-}\right.$radiation), single-crystal x-ray (Bruker, Mo K $\alpha_{-}$radiation), Laue diffraction (Photonic Science), and scanning electron microscope (ZEISS GeminiSEM 500)] with an energy dispersive x-ray spectroscopy probe (EDX). Main physical characterization (resistivity and specific heat) was performed using a Physical Property Measurement System (PPMS), showing a transition at $323 \mathrm{~K}$.

High-pressure Raman studies were performed up to $10 \mathrm{GPa}$ at room temperature using a Mao-Bell-type diamond anvil cell (DAC). A very small piece from the bulk crystal $(\approx 30 \mu \mathrm{m})$, along with a very small ruby chip $(\approx 10 \mu \mathrm{m})$ were placed inside a hole of diameter $\approx 200 \mu \mathrm{m}$ in a stainless steel gasket kept between the two diamonds of the DAC. A methanol-ethanol-water (16:3:1 ratio) mixture was used as a pressure-transmitting medium. The ruby fluorescence method [20] was used for pressure calibration. Raman spectra were recorded in a LabRam spectrometer $(\mathrm{M} / \mathrm{s}$ Horiba) in back-scattering geometry, using $50 \times$ objective and laser excitation of $532 \mathrm{~nm}$ from a diode-pumped solid state laser.

\section{FIRST-PRINCIPLES COMPUTATIONAL DETAILS}

Our first-principles calculations are based on the density functional theory as implemented in the QUANTUM ESPRESSO package [21], modeling the interaction between ionic cores and valence electrons with ultrasoft pseudopotentials [22]. The exchange-correlation energy of electrons is treated within a generalized gradient approximation (GGA) and Perdew-Bruke-Ernzerhof (PBE) functional form [23]. We use energy cutoffs of 40 and 320 Ry to truncate the planewave basis used to represent Kohn-Sham wave functions and charge density respectively. Self-consistent solution to the Kohn-Sham equations was obtained until the total energy converges numerically to less than $10^{-8}$ Ry. Structures were relaxed to minimize the energy until the magnitude of Hellman-Feynman force on each atom is less than 0.001 Ry/bohr. We included the van der Waals (vdW) interaction with parametrization given in the Grimme scheme [24]. In self-consistent Kohn-Sham (KS) calculations of structural configurations of $\mathrm{Ta}_{2} \mathrm{NiSe}_{5}$ with monoclinic $(C 2 / c)$ and orthorhombic $(\mathrm{Cmcm})$ unit cells, Brillouin zone (BZ) integrations were sampled on a uniform mesh of $10 \times 10 \times 4 \mathbf{k}$ points and on $10 \times 6 \times 2$ uniform mesh of $\mathbf{k}$ points in the Brillouin zone of orthorhombic (Pmnm) unit cells.

Since DFT typically underestimates the band gap and the $d$ states of Ni and Ta are partially filled, we included on-site electron correlations modeled with Hubbard U parameters. We determined dynamical matrix and phonon spectrum at the $\Gamma$ point using a frozen phonon method with atomic displacements of $\pm 0.04 \AA$ in each Cartesian direction. 

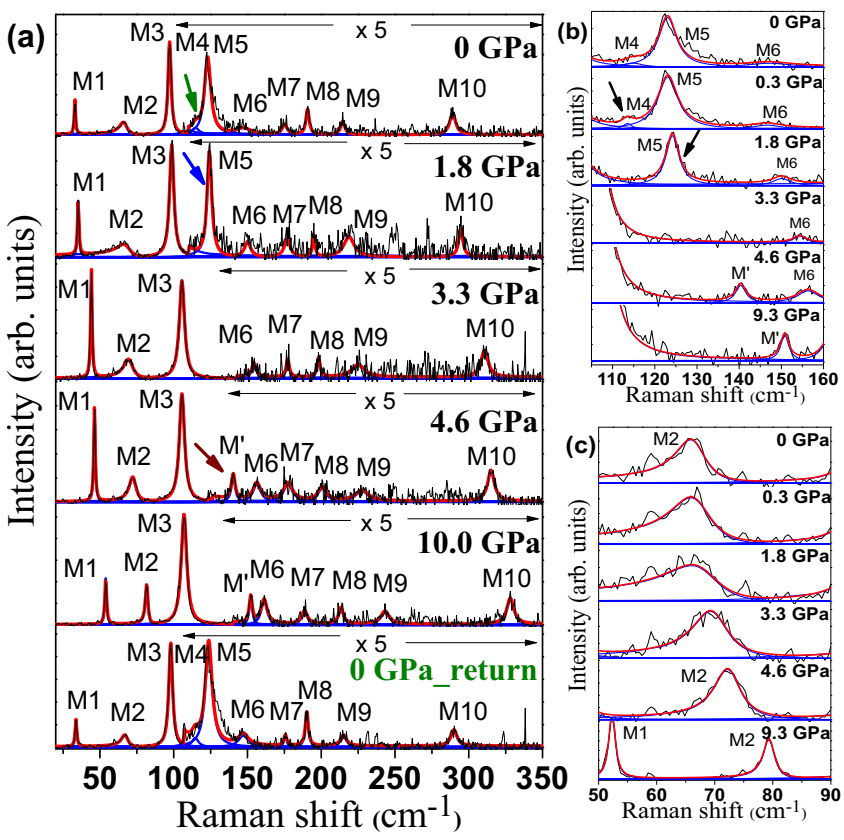

FIG. 1. Pressure evolution of Raman spectra. Solid lines (red and blue) are the Lorentzian fits to experimental data points (black). Appearance of new modes is indicated by arrows.

\section{RESULTS AND DISCUSSION}

\section{A. Experiments}

High-pressure Raman experiments on $\mathrm{Ta}_{2} \mathrm{NiSe}_{5}$ were carried out at room temperature. We observe 10 Raman modes at ambient pressure [shown in the top panel of Fig. 1(a)] marked as M1 $\left(33.1 \mathrm{~cm}^{-1}\right)$, M2 $\left(66.9 \mathrm{~cm}^{-1}\right)$, M3 $\left(97.3 \mathrm{~cm}^{-1}\right), \mathrm{M} 4$ $\left(113.6 \mathrm{~cm}^{-1}\right)$, M5 $\left(122.8 \mathrm{~cm}^{-1}\right)$, M6 $\left(146.7 \mathrm{~cm}^{-1}\right)$, M7 $(175.5$ $\left.\mathrm{cm}^{-1}\right)$, M8 (191.2 $\left.\mathrm{cm}^{-1}\right)$, M9 $\left(214.9 \mathrm{~cm}^{-1}\right)$, and M10 (289.2 $\left.\mathrm{cm}^{-1}\right)$. The symmetry assignments of these modes have been done by Werdehausen et al. [11]. Raman spectra of TNSe at a few pressures displayed in Fig. 1(a) show the following features: M4 phonon mode (marked by green arrow) disappears around $\approx 1.0 \mathrm{GPa}$. The disappearance of mode M5 (marked by the blue arrow) takes place around $\approx 3.0 \mathrm{GPa}$, and a new mode $\mathrm{M}^{\prime}$ (marked by brown arrow) appears at $130 \mathrm{~cm}^{-1}$ beyond 3.8 GPa. These features are clearly shown in the expanded region of $105-160 \mathrm{~cm}^{-1}$ at a few typical pressures in Fig. 1(b). Raman spectra in the decreasing pressure cycle show that the transitions are reversible.

A noticeable asymmetric line shape, known as the BreitWigner-Fano (BWF) line, is observed for the Raman mode M2, and its evolution at a few characteristic pressures is shown in Fig. 1(c). The asymmetric BWF line shape seen in graphene [25-27], doped semiconducters like GaAs [28], doped silicon [29,30], and metals [31,32] arises from the interference between the Raman amplitudes of a discrete state (like phonons) and continuum of excitations [33] (like electronic continuum in metals and excitonic continuum in TNSe [14]). It is given by $I(\omega)=I_{0} \frac{\left[1+\left(\omega-\omega_{0}\right) / q \Gamma\right]^{2}}{1+\left[\left(\omega-\omega_{0}\right) / \Gamma\right]^{2}}$, where $|1 / q|$ is an asymmetry parameter characterizing the strength of coupling between the discrete state and the continuum of states, and $\Gamma$ and $\omega_{0}$ are the broadening parameter and uncoupled phonon

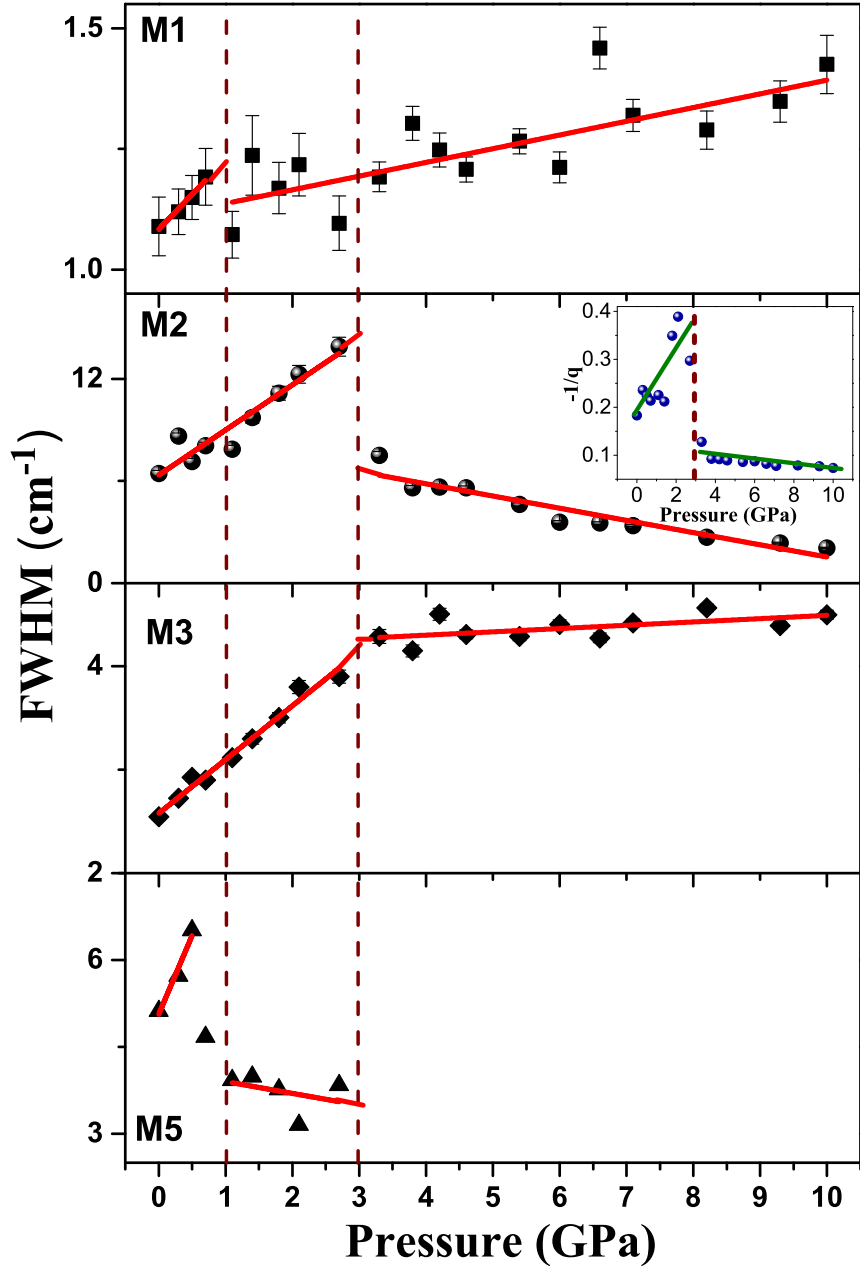

FIG. 2. Pressure dependence of line widths of the strong Raman modes of $\mathrm{Ta}_{2} \mathrm{NiSe}_{5}$. Red solid lines are linear fit to the data. The phase transitions around 1.0 and $3.0 \mathrm{GPa}$ are marked by the brown dashed line where FWHM shows change in the linear slope for modes M1 and M5. The inset figure shows the variation of the asymmetry parameter $|1 / q|$ with pressure. The green lines are guide to the eyes.

frequency, respectively [34]. The pressure dependence of $\Gamma$ is shown in Fig. 2 along with the line widths of some of the strong modes as a function of pressure; red solid lines are linear fits to the data and the dashed lines at $\approx 1.0$ and $\approx 3.0 \mathrm{GPa}$ indicate the transition pressure values. The inset figure shows the variation of the Fano asymmetry parameter $|1 / q|$ of the mode M2 with pressure. The $q$ parameter is defined by $q=$ $\frac{1}{\pi D_{e-h}\left(E_{\Omega}\right) V_{e-p h}} \frac{\mu_{p h}}{\mu_{e-h}}$, where $D_{e-h}\left(E_{\Omega}\right)$ is the joint electron-hole pair density of states at the renormalized phonon energy, $V_{e-p h}$ is the electron-phonon coupling strength, and $\mu_{p h}$ and $\mu_{e-h}$ are the optical matrix elements of the dressed phonon and that of an electron hole pair respectively [27]. Smaller values of $q$ correlate with higher asymmetry. The band gap reduces with increasing pressure, resulting in an increase in the coupling strength $1 / q$ within the semiconducting phase, and above that it decreases drastically at $P_{c 2} \approx 3.0 \mathrm{GPa}$ and goes toward zero when the system undergoes a semiconductor to semimetal transition. This happens due to the reduction of 

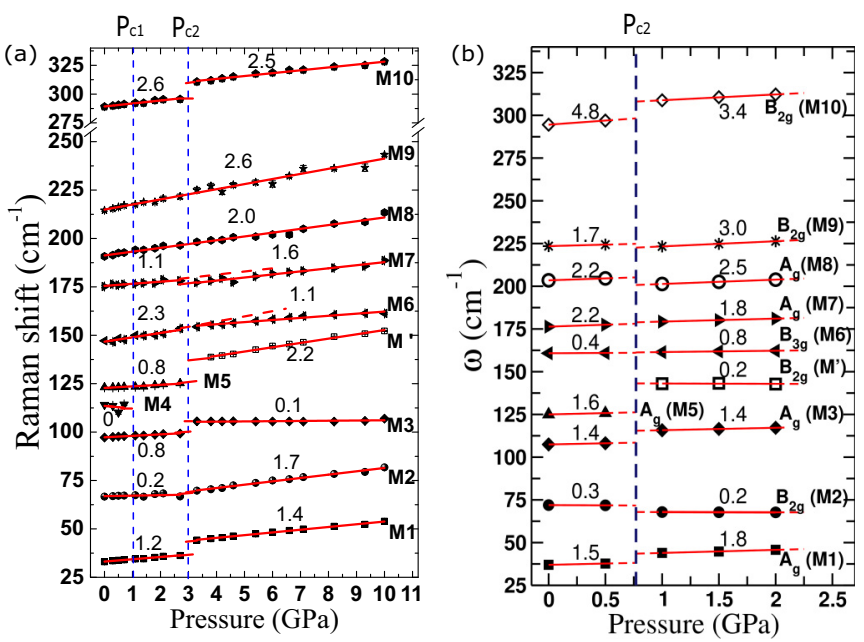

FIG. 3. (a) Raman shift vs pressure plot. Solid red lines are linear fits to the observed frequencies. The numbers next to the straight lines are the fitted value of $d \omega / d P$ in $\mathrm{cm}^{-1} / \mathrm{GPa}$. The blue dashed line marks the phase transition at $P_{c 1}=1 \mathrm{GPa}$ and $P_{c 2}=3 \mathrm{GPa}$. (b) Theoretically calculated variation in frequency of Raman active modes with pressure. The red lines are linear fits to the observed frequencies (solid symbols) and the corresponding slope values are given (in $\mathrm{cm}^{-1} / \mathrm{GPa}$ ). The vertical blue dashed line marks the phase transition $P_{c 2}$.

electronic transition in the semimetallic phase as a result of Pauli blocking [27]. The following observations can be made from Fig. 2: (i) Electron-phonon coupling increases with pressure up to $P_{c 1}$ for M1 and M5 modes and up to $P_{c 2}$ for M2 and M3 modes. (ii) Electron-phonon coupling does not vary much beyond $P_{c 2}$.

Pressure dependence of the mode frequencies as extracted from Lorentzian fits (except for mode M2, which has been fitted to BWF line shape) to the spectra and [Fig. 3(a)] linear fitted (solid lines) to $\omega=\omega_{0}+(d \omega / d P) P$. The values of $\omega_{0}$ and the pressure derivative $d \omega / d P$ are listed in Table 1 for all the phases. The following observations can be made from Fig. 3(a): (i) Mode M4 disappears above $P_{c 1} \approx 1.0 \mathrm{GPa}$, (ii) mode M5 disappears above $P_{c 2} \approx 3.0 \mathrm{GPa}$, (iii) a new mode $\mathrm{M}^{\prime}$ appears above $P_{c 2}$, and (iv) all the modes except M8 and M9 show a change in slope at $\approx 3.0 \mathrm{GPa}$. The first observation, namely the absence of M4 mode, marks a phase transition from monoclinic $C 2 / c$ (phase I) to orthorhombic $\mathrm{Cmcm}$ (phase II) as seen in high-pressure x-ray diffraction studies [18]. Following Ref. [18], the transition at $P_{c 2}$ is associated with transition from orthorhombic $\mathrm{Cmcm}$ (phase II) to primitive orthorhombic Pmnm (phase III).

\section{B. Theoretical calculations}

We now present results of first-principles DFT-based calculations of electronic structure and phonons to examine the phase transition at $P_{c 2}$. Electronic structure of the orthorhombic structure of $\mathrm{Ta}_{2} \mathrm{NiSe}_{5}$ with $\mathrm{Cmcm}$ space group [Fig. 4(a)] shows that it is metallic when calculated within a GGA-based semilocal DFT. As the splitting of bands due to spin-orbit coupling (SOC) is relatively small, we have not included SOC in our pressure-dependent calculations. Second, the standard
TABLE I. Pressure coefficients and Grüneisen parameters for various modes of $\mathrm{Ta}_{2} \mathrm{NiSe}_{5}$ observed for various phases.

\begin{tabular}{|c|c|c|c|c|}
\hline Phase & Mode & $\begin{array}{c}\text { Mode frequency } \\
\qquad\left(\omega_{0}\right) \\
\left(\mathrm{cm}^{-1}\right)\end{array}$ & $\begin{array}{c}d \omega / d P \\
\left(\mathrm{~cm}^{-1} / \mathrm{GPa}\right)\end{array}$ & $\gamma_{i}$ \\
\hline Phase I & M1 $\left(\mathrm{A}_{g}\right)$ & $33.1 \pm 0.1$ & $1.2 \pm 0.1$ & 1.6 \\
\hline$(C 2 / c)$ & $\mathrm{M} 2\left(\mathrm{~B}_{1 g}\right)$ & $66.9 \pm 0.3$ & $0.2 \pm 0.2$ & 0.13 \\
\hline 0 to 1.0 & M3 $\left(\mathrm{B}_{1 g}\right)$ & $97.3 \pm 0.1$ & $0.8 \pm 0.1$ & 0.35 \\
\hline \multirow[t]{7}{*}{$\mathrm{GPa}$} & M4 ( $\left.\mathrm{B}_{1 g}\right)$ & $113.6 \pm 2.0$ & $\approx 0$ & \\
\hline & $\operatorname{M5}\left(\mathrm{A}_{g}\right)$ & $122.8 \pm 0.2$ & $0.8 \pm 0.1$ & 0.29 \\
\hline & M6 $\left(\mathrm{A}_{g}\right)$ & $146.7 \pm 0.4$ & $2.3 \pm 0.3$ & 0.68 \\
\hline & M7 $\left(\mathrm{A}_{g}\right)$ & $175.5 \pm 0.3$ & $1.1 \pm 0.2$ & 0.28 \\
\hline & $\operatorname{M} 8\left(\mathrm{~B}_{1 g}\right)$ & $191.2 \pm 0.3$ & $2.0 \pm 0.1$ & 0.46 \\
\hline & M9 ( $\left.\mathrm{B}_{1 g}\right)$ & $214.9 \pm 0.5$ & $2.6 \pm 0.1$ & 0.54 \\
\hline & $\operatorname{M10}\left(\mathrm{B}_{1 g}\right)$ & $289.2 \pm 0.3$ & $2.6 \pm 0.2$ & 0.40 \\
\hline Phase III & M1 & $39.7 \pm 0.2$ & $1.4 \pm 0.1$ & 1.7 \\
\hline (Pmnm) & M2 & $64.2 \pm 0.4$ & $1.7 \pm 0.1$ & 1.3 \\
\hline 3.0 to 10.0 & M3 & $104.9 \pm 0.4$ & $0.1 \pm 0.1$ & 0.05 \\
\hline \multirow[t]{6}{*}{$\mathrm{GPa}$} & $M^{\prime}$ (new) & $130.5 \pm 0.5$ & $2.2 \pm 0.1$ & 0.81 \\
\hline & M6 & $151.4 \pm 0.5$ & $1.1 \pm 0.1$ & 0.35 \\
\hline & M7 & $171.9 \pm 0.8$ & $1.6 \pm 0.1$ & 0.45 \\
\hline & M8 $\left(\mathrm{B}_{1 g}\right)$ & $191.2 \pm 0.3$ & $2.0 \pm 0.1$ & 0.48 \\
\hline & M9 ( $\left.\mathrm{B}_{1 g}\right)$ & $214.9 \pm 0.5$ & $2.6 \pm 0.1$ & 0.54 \\
\hline & M10 & $303.5 \pm 0.5$ & $2.5 \pm 0.1$ & 0.40 \\
\hline
\end{tabular}

DFT-based electronic structure calculations typically underestimate the band gap, and hence we determined electronic structure of $\mathrm{Ta}_{2} \mathrm{NiSe}_{5}$ using SCAN meta-GGA [35] exchange correlation energy functional as implemented in the VASP code and find that it is still metallic (Fig. 5). Thus, the SCAN meta-GGA-based DFT description is also unable to capture the semiconducting electronic structure of the $\mathrm{Cmcm}$
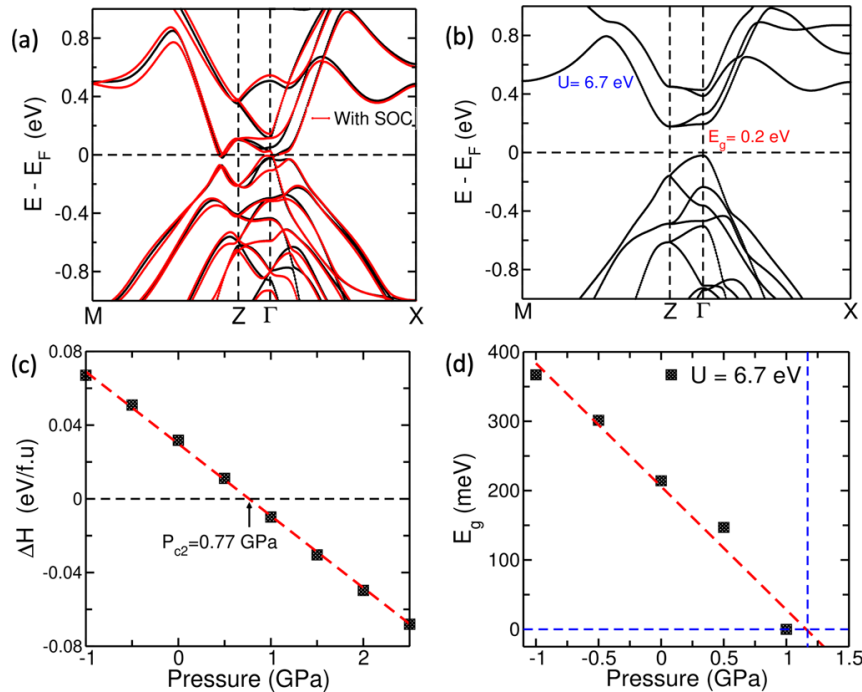

FIG. 4. (a) Electronic structure of $\mathrm{Ta}_{2} \mathrm{NiSe}_{5}$ in orthorhombic $\mathrm{Cmcm}$ phase determined with (red color lines) and without (black color lines) effects of SOC, (b) Electronic structure of orthorhombic (Cmcm) phase with $U=6.7 \mathrm{eV}$, (c) difference in enthalpy of $\mathrm{Cmcm}$ and Pmnm phases of $\mathrm{Ta}_{2} \mathrm{NiSe}_{5}$ for $U=6.7 \mathrm{eV}$ at $P=0 \mathrm{GPa}$, and (d) band gap of $\mathrm{Cmcm}$ phase as a function of hydrostatic pressure. 


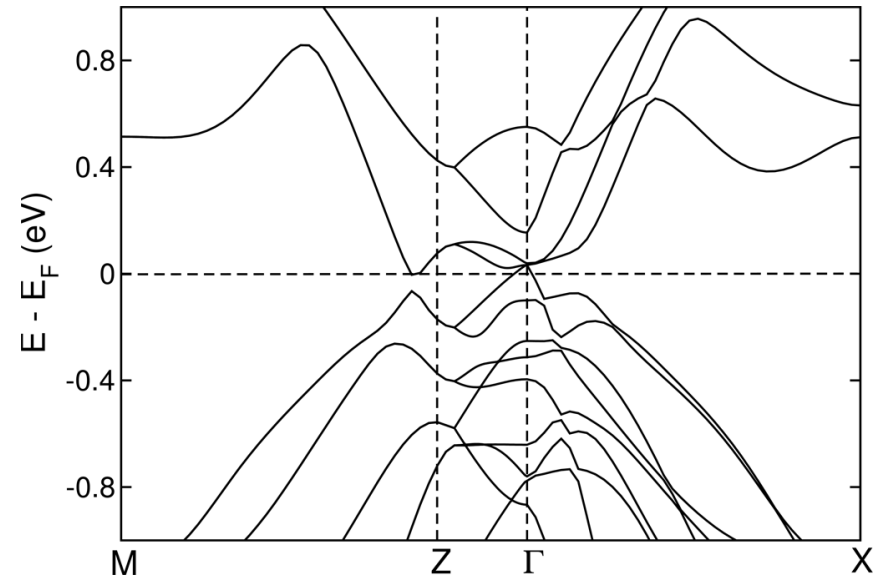

FIG. 5. Electronic structure of $\mathrm{Ta}_{2} \mathrm{NiSe}_{5}$ in orthorhombic $\mathrm{Cmcm}$ phase determined using SCAN meta-GGA exchange correlation functional as implemented in VASP code.

phase. To have a model description that is qualitatively correct with a nonzero gap, we thus consider including Hubbard $U$ parametrized on-site electron correlations of Ta $5 d$ orbitals and find that a gap opens up at $U \geqslant 6.4 \mathrm{eV}, E_{g}=44.6 \mathrm{meV}$ when $U=6.4 \mathrm{eV}$, in reasonable agreement with experiments $\left(E_{g}^{e x p}<50 \mathrm{meV}\right)$ [6]. Indeed, the electronic band gap of TNSe depends sensitively on $U$, pressure, and structure. We carried out a series of test calculations of electronic structure varying the value of $U$, and chose to work with $U=6.7 \mathrm{eV}$, which gives a direct band gap at $\Gamma$ point of the $C \mathrm{mcm}$ phase to be to be $\approx 0.2 \mathrm{eV}$ at $P=0$ [Fig. 4(b)] and remains finite for $P<$ 1 GPa [Fig. 4(d)].

To investigate the pressure-dependent phase transition from orthorhombic Cmcm to Pmnm phase, we estimated the changes in enthalpy $\Delta H\left(=H_{P m n m}-H_{C m c m}\right)$, which decreases with pressure and attains a negative value at $0.77 \mathrm{GPa}$ [Fig. 4(c)] in calculations with $U=6.7 \mathrm{eV}$. Our estimate of the transition pressure $P_{c 2}$ is $0.77 \mathrm{GPa}$. Further analysis of the evolution of electronic structure with hydrostatic pressure shows (i) reduction in $E_{g}$ with $P$ [Fig. 4(d)] and (ii) the electronic structure near the gap is highly sensitive to pressure as well as Hubbard $U$ (see Fig. 6). The discrepancy between the observed $P_{c 2}(3 \mathrm{GPa})$ and calculated $P_{c 2}(0.77 \mathrm{GPa})$ is partly due to errors in the calculated equilibrium lattice constants and their strong sensitivity to the $U$ term needed to capture electronic structure accurately.

The calculated values of Raman frequencies in $\mathrm{Cmcm}$ (below $0.77 \mathrm{GPa}$ ) and Pmnm (above $0.77 \mathrm{GPa}$ ) phases are shown in Fig. 3(b), which shows a clear phase transition at theoretical $P_{c 2}=0.77 \mathrm{GPa}$. A careful examination of the evolution of electronic structure with pressure shows the pressure-induced semiconductor $(\mathrm{Cmcm})$ to semimetal $(\mathrm{Pmnm})$ transition at $0.77 \mathrm{GPa}$ [Figs. 7 and 4(c)]. As shown in Fig. 3(b), the structural phase transition from orthorhombic $\mathrm{Cmcm}$ to Pmnm is marked by disappearance of the $A_{g}$ Raman mode at $126 \mathrm{~cm}^{-1}$ above $0.77 \mathrm{GPa}$ and appearance of a new $B_{2 g}$ Raman mode at $143 \mathrm{~cm}^{-1}$, in addition to the changes in pressure coefficients $(d \omega / d P)$ of Raman modes, which are in good agreement with our experimental results [Fig. 3(a)]. Thus, there is a clear correlation between the slope change of Raman

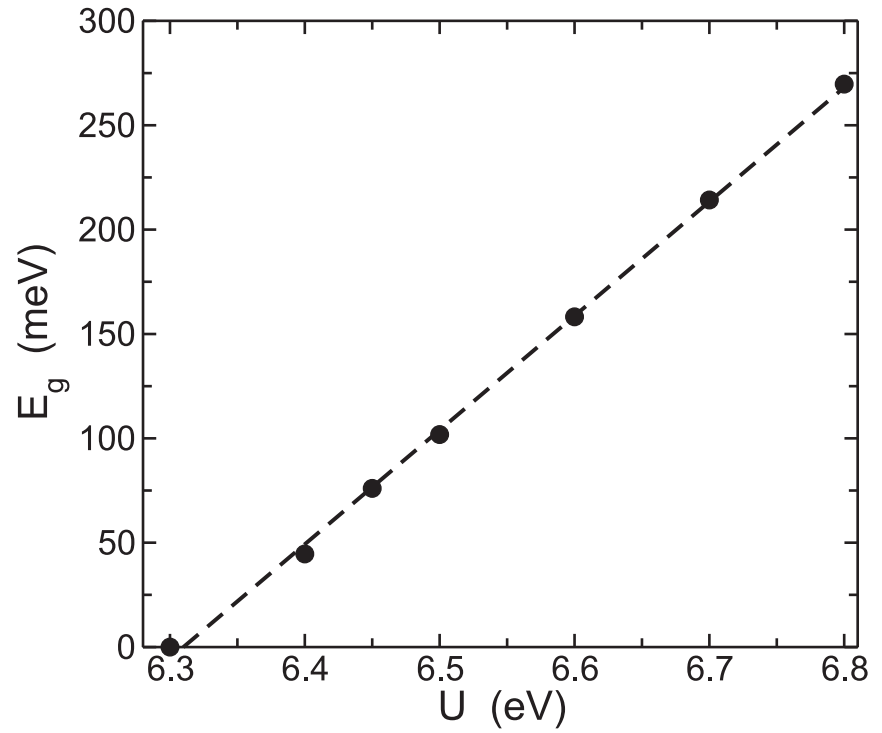

FIG. 6. Band gap of the semiconducting $\mathrm{Cmcm}$ phase as a function of the $U$ parameter showing strong sensitivity of the electronic structure of $\mathrm{Ta}_{2} \mathrm{NiSe}_{5}$ to the Hubbard $U$ term.

active modes and structural transition accompanying the electronic phase transition, obtained within the same theoretical framework.

Since DFT calculations do not correctly capture the excitonic insulator ground state, we develop a simple model of phonons coupled with the frontier electronic states and demonstrate how strong electron-phonon coupling drives a pressure-dependent structural transition $\left(P_{c 1}\right)$ concurrent with the change from excitonic to normal semiconducting electronic structure. We consider the coupling of the $v$ phonon with an excitonic state involving conduction band $|C\rangle$ and valence band $|V\rangle$, and express it using Hellmann-Feynman
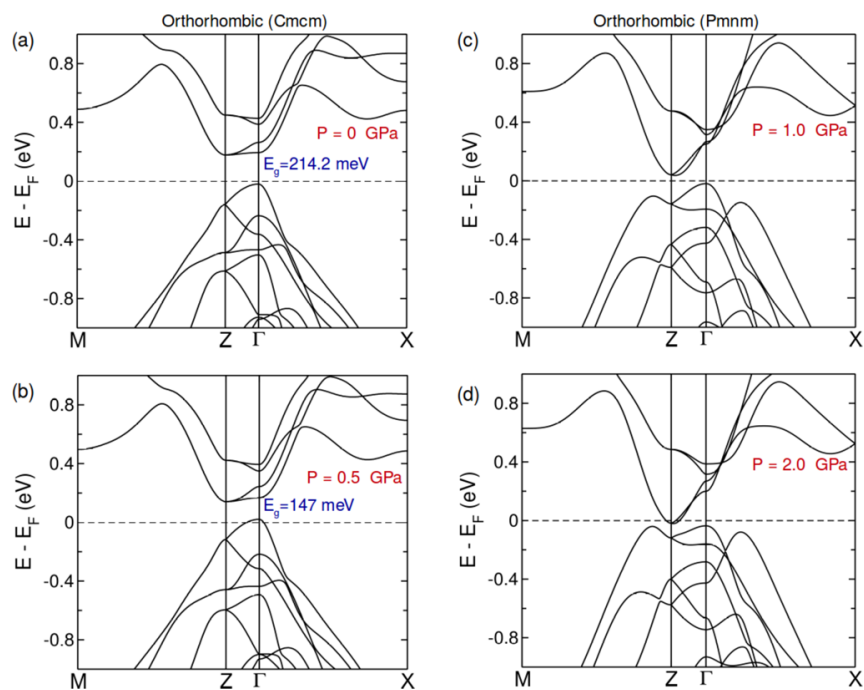

FIG. 7. Electronic structures of $\mathrm{Ta}_{2} \mathrm{NiSe}_{5}$ in orthorhombic phase at (a) $0 \mathrm{GPa}(\mathrm{Cmcm})$, (b) $0.5 \mathrm{GPa}(\mathrm{Cmcm})$, (c) $1.0 \mathrm{GPa}($ Pmnm), (d) $2.0 \mathrm{GPa}(P m n m)$ showing a pressure induced semiconductor to semimetal transition from orthorhombic $\mathrm{Cmcm}$ to $P$ mnm above $P_{c}=$ $0.77 \mathrm{GPa}$. 
theorem as the force projected on to eigenvector of phonon $v: f_{c}\left\langle C\left|\Delta V_{v}\right| C\right\rangle+f_{v}\left\langle V\left|\Delta V_{v}\right| V\right\rangle+F_{0}=\vec{F} \cdot \hat{e}_{v}=F_{\nu}$, where $F_{0}$ is the total contribution $2 \Sigma_{i}\left\langle i\left|\Delta V_{v}\right| i\right\rangle$ of electronic states with energies below that of $|V\rangle, \Delta V_{v}$ is the phonon perturbing potential, and $f_{c}$ and $f_{v}$ are occupation numbers of states $|C\rangle$ and $|V\rangle$. Components of $\vec{F}$ are the forces acting on atoms as a result of formation of an electron-hole pair.

To determine $F_{0}$, we consider the normal semiconducting (gapped) ground state with $f_{v}=2$ and $f_{c}=0$. Noting that force is zero in the equilibrium (relaxed) structure, we get $2\left\langle V\left|\Delta V_{v}\right| V\right\rangle+F_{0}=0$. We use a self-consistent Kohn-Sham calculation of a supercell with fixed occupation numbers of states at $\Gamma$ point to model excitation of electrons from the VBM to CBM such that $f_{v}=2-f_{c}$. This gives $f_{c}\left[\left\langle C\left|\Delta V_{v}\right| C\right\rangle-\left\langle V\left|\Delta V_{v}\right| V\right\rangle\right]=F_{\nu}$. Thus, $F_{v}$ is the coupling of phonon $v$ with exciton defined by the difference in coupling of a phonon with electron and hole in states $|C\rangle$ and $|V\rangle$ respectively. We obtain $F_{v}$ as the projection of forces exerted on atoms (due to formation of exciton at the $\Gamma$ point) onto the eigenvector of phonon $v$. In a simple model of phonon (amplitude $u_{v}$ ) coupled with $|C\rangle$ and $|V\rangle$, the total energy of a state characterized by $f_{c} \neq 0$ is

$$
E=f_{c}\left(\epsilon_{c}-\epsilon_{v}\right)+2 \epsilon_{v}+\frac{1}{2} K_{v} u_{v}^{2}+f_{c} F_{v} u_{v},
$$

where $K_{v}$ is the effective spring constant of phonon $v$ with amplitude $u_{v}$. The ground state of this model is determined by a minimum of energy $E\left(u_{\min }^{v}=-\frac{f_{c} F_{v}}{K}\right)$, which gives the change in atomic structure (projected onto the phonon $v$ ) due to formation of electron-hole pairs and consequent renormalized energy is

$$
E=f_{c}\left(\epsilon_{c}-\epsilon_{v}\right)+2 \epsilon_{v}-\frac{f_{c}^{2} F_{v}^{2}}{2 K_{v}},
$$

where the first term gives electronic energy cost to create an exciton ( $\epsilon_{v}$ can be set to zero) and the last term represents gain in energy due to renormalization by phonon $v$. For an EI state to exist, $E$ should be negative. For $\left(0<f_{c}<1\right), \epsilon_{v}=0$, a solution of $E=0$ exists for $f_{c}<1\left(f_{c}=0\right.$ being the normal state). This is possible when

$$
\left(\epsilon_{c}-\epsilon_{v}\right)<\frac{F_{v}^{2}}{2 K_{v}} .
$$

The impact of the exciton-phonon coupling is determined by an energy scale that is geometric mean of the energy of phonon and electronic band gap. A large value of $F_{v}$ means that the exciton-phonon coupling is large and a small value of the interatomic force constant $\left(\mathrm{K}_{v}\right)$ means that the phonon $v$ is soft. Thus, a soft phonon mode coupling strongly with electron-hole pair contributes significantly to the stability of an excitonic insulating state.

To find applicability of this mechanism to the EI phase in $\mathrm{Ta}_{2} \mathrm{NiSe}_{5}$, we determined all its parameters from first principles. We simulated $4 \times 1 \times 1$ supercell of monoclinic $\mathrm{Ta}_{2} \mathrm{NiSe}_{5}$ with $\Gamma$ point sampling and determined the forces exerted on atoms after exciting an electron from VBM to CBM. Our estimate of the exciton formation energy, i.e., the energy required to remove an electron from valence band and introduce it in the CBM (forming an electron-hole pair), is $0.22 \mathrm{eV}$, which is comparable to the optical excitation gap
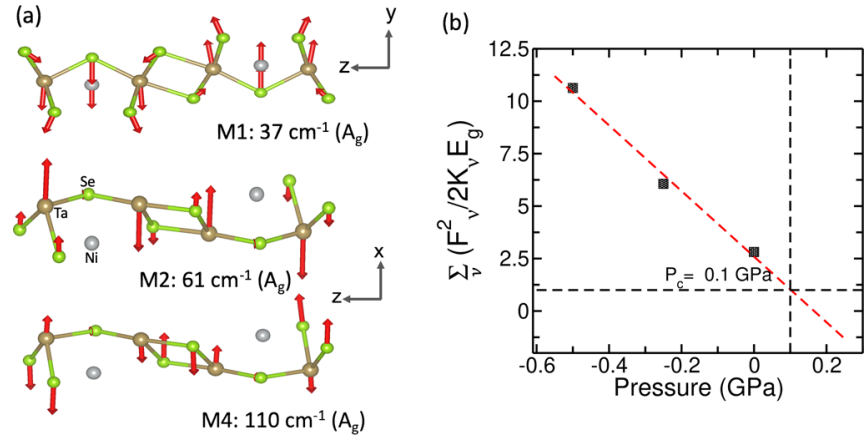

FIG. 8. (a) Atomic displacements of soft Raman active phonons, which couple strongly with excitons in $\mathrm{Ta}_{2} \mathrm{NiSe}_{5}$ and (b) $\sum_{v}\left(F_{v}^{2} / 2 K_{v} E_{g}\right)$ as a function of pressure $P(\mathrm{GPa})$.

$(\approx 0.16 \mathrm{eV})$ [6]. From overlaps between phonon eigenvector $\left(\hat{e}_{v}\right)$ and the forces exerted on atoms due to formation of electron-hole pair, we estimated $F_{v}$, the exciton-phonon coupling. We find that the Raman active modes satisfy condition in Eq. (3), confirming that this mechanism can explain the stability of excitonic insulator state in $\mathrm{Ta}_{2} \mathrm{NiSe}_{5}$ at $P=0 \mathrm{GPa}$. The eigenvectors of these soft $A_{g}$ Raman modes [Fig. 8(a)] involve out of phase displacement of $\mathrm{Ta}$ and $\mathrm{Ni}$-Se chains (along the $a$ axis). This is the phonon reported to be strongly coupled with the exciton condensate in Ref. [11]. With parameters $\left(F_{v}\right.$ and $\left.K_{v}\right)$ obtained as a function of pressure from our first-principles calculations, we plot $\sum_{v}\left(F_{v}^{2} / 2 K_{v} E_{g}\right)$ versus pressure in Fig. 8(b). Thus, our model captures the essential aspects of excitonic insulator to normal semiconductor phase transition at $P_{c 1} \approx 0.1 \mathrm{GPa}$, uncovering the role of $A_{g}$ Raman modes in the transition.

\section{CONCLUSIONS}

In conclusion, we have uncovered Raman anomalies occurring at a pressure-induced excitonic insulator to semiconductor transition in $\mathrm{Ta}_{2} \mathrm{NiSe}_{5}$. A combination of experiments, first-principles calculations of the exciton-phonon coupling, and theoretical analysis of the model Hamiltonian presented here shows that a strong coupling of excitons with soft Raman active modes is adequate to stabilize its EI phase. Our work thus supports the BCS-like mechanism of preformed excitons hinted at recently by Lee et al. [17] and Larkin et al. [14,16], which condense into an EI state below $T_{c}$, rather than a purely strong coupling between electrons and holes. It also corroborates observations of phonon-exciton complexes in vibrational spectroscopic experiments [11,14]. Another structural transition marked by the disappearance of $A_{g}$ Raman mode at $123 \mathrm{~cm}^{-1}$ and appearance of a new $B_{2 g}$ Raman mode at $130 \mathrm{~cm}^{-1}$ (above $3 \mathrm{GPa}$ in experiments) is identified as a pressure-induced semiconductor to semimetal phase transition by first-principles calculations.

\section{ACKNOWLEDGMENTS}

A.K.S. acknowledges Department of Science and Technology (DST) Nanomission Council and Year of Science Professorship for financial support. S.P. acknowledges DST for 
support through an Inspire fellowship. U.V.W. acknowledges support from the J.C. Bose National Fellowship and a project funded by IKST. S.G. is thankful to Jawaharlal Nehru Centre for Advanced Scientific Research, India, for a research fellowship and Thematic Unit of Excellence on Computational
Material Science, JNCASR, India, for computational resources. L.H. acknowledges DST, India [Grant No. SR/WOSA/PM-33/2018 (G)] for funding support and Dr. Surjeet Singh for allowing the use of crystal growth facilities in his laboratory.
[1] D. Jérome, T. M. Rice, and W. Kohn, Phys. Rev. 158, 462 (1967).

[2] W. Kohn, Phys. Rev. Lett. 19, 439 (1967).

[3] B. I. Halperin and T. M. Rice, Rev. Mod. Phys. 40, 755 (1968).

[4] J. Kuně̌, J. Condens. Matter Phys. 27, 333201 (2015).

[5] S. A. Sunshine and J. A. Ibers, Inorg. Chem. 24, 3611 (1985).

[6] Y. F. Lu, H. Kono, T. I. Larkin, A. W. Rost, T. Takayama, A. V. Boris, B. Keimer, and H. Takagi, Nat. Commun. 8, 14408 (2017).

[7] F. J. Di Salvo, C. H. Chen, R. M. Fleming, J. V. Waszczak, R. G. Dunn, S. A. Sunshine, and J. A. Ibers, J. Less Common Met. 116, 51 (1986).

[8] Y. Wakisaka, T. Sudayama, K. Takubo, T. Mizokawa, M. Arita, H. Namatame, M. Taniguchi, N. Katayama, M. Nohara, and H. Takagi, Phys. Rev. Lett. 103, 026402 (2009).

[9] Y. Wakisaka, T. Sudayama, K. Takubo, T. Mizokawa, N. L. Saini, M. Arita, H. Namatame, M. Taniguchi, N. Katayama, M. Nohara, and H. Takagi, J. Supercond. Nov. Magn. 25, 1231 (2012).

[10] K. Mu, H. Chen, Y. Li, Y. Zhang, P. Wang, B. Zhang, Y. Liu, G. Zhang, L. Song, and Z. Sun, J. Mater. Chem. C 6, 3976 (2018).

[11] D. Werdehausen, T. Takayama, M. Höppner, G. Albrecht, A. W. Rost, Y. Lu, D. Manske, H. Takagi, and S. Kaiser, Sci. Adv. 4, eaap8652 (2018).

[12] T. Kaneko, T. Toriyama, T. Konishi, and Y. Ohta, Phys. Rev. B 87, 035121 (2013).

[13] K. Sugimoto, S. Nishimoto, T. Kaneko, and Y. Ohta, Phys. Rev. Lett. 120, 247602 (2018).

[14] T. I. Larkin, A. N. Yaresko, D. Pröpper, K. A. Kikoin, Y. F. Lu, T. Takayama, Y.-L. Mathis, A. W. Rost, H. Takagi, B. Keimer, and A. V. Boris, Phys. Rev. B 95, 195144 (2017).

[15] A. Nakano, T. Hasegawa, S. Tamura, N. Katayama, S. Tsutsui, and H. Sawa, Phys. Rev. B 98, 045139 (2018).

[16] T. I. Larkin, R. D. Dawson, M. Höpner, T. Takayama, M. Isobe, Y.-L. Mathis, H. Takagi, B. Keimer, and A. V. Boris, Phys. Rev. B 98, 125113 (2018).

[17] J. Lee, C.-J. Kang, M. J. Eom, J. S. Kim, B. I. Min, and H. W. Yeom, Phys. Rev. B 99, 075408 (2019).
[18] A. Nakano, K. Sugawara, S. Tamura, N. Katayama, K. Matsubayashi, T. Okada, Y. Uwatoko, K. Munakata, A. Nakao, H. Sagayama, R. Kumai, K. Sugimoto, N. Maejima, A. Machida, T. Watanuki, and H. Sawa, IUCrJ 5, 158 (2018).

[19] L. M. Falicov and J. C. Kimball, Phys. Rev. Lett. 22, 997 (1969).

[20] H. K. Mao, J. Xu, and P. M. Bell, J. Geophys. Res. Solid Earth 91, 4673 (1986).

[21] P. Giannozzi, S. Baroni, N. Bonini, M. Calandra, R. Car, C. Cavazzoni, D. Ceresoli, G. L. Chiarotti, M. Cococcioni, I. Dabo, A. Dal Corso, S. de Gironcoli, S. Fabris, G. Fratesi, R. Gebauer, U. Gerstmann, C. Gougoussis, A. Kokalj, M. Lazzeri, L. Martin-Samos et al., J. Phys.: Condens. Matter 21, 395502 (2009).

[22] D. Vanderbilt, Phys. Rev. B 41, 7892 (1990).

[23] J. P. Perdew, K. Burke and M. Ernzerhof, Phys. Rev. Lett. 77, 3865 (1996).

[24] S. Grimme, J. Comput. Chem. 27, 1787 (2006).

[25] D. H Chae, T. Utikal, S. Weisenburger, H. Giessen, K. v. Klitzing, M. Lippitz, and J. Smet, Nano Lett. 11, 1379 (2011).

[26] D. Yoon, D. Jeong, H. J. Lee, R. Saito, Y. W. Son, H. Cheol Lee, and H. Cheong, Carbon 61, 373 (2013).

[27] T. T. Tang, Y. Zhang, C. H. Park, B. Geng, C. Girit, Z. Hao, M. C. Martin, A. Zettl, M. F. Crommie, S. G. Louie, Y. R. Shen, and F. Wang, Nat. Nanotechnol. 5, 32 (2009).

[28] M. Vinod, G. Raghavan, and V. Sivasubramanian, Sci. Rep. 8 , 17706 (2018).

[29] F. Cerdeira, T. A. Fjeldly, and M. Cardona, Phys. Rev. B 8, 4734 (1973).

[30] N. H. Nickel, P. Lengsfeld, and I. Sieber, Phys. Rev. B 61, 15558 (2000).

[31] V. G. Sathe, S. Tyagi, and G. Sharma, J. Phys. Conf. 755 , 012008 (2016).

[32] E. H. Hasdeo, A. R. T. Nugraha, K. Sato, M. S. Dresselhaus, and R. Saito, Phys. Rev. B 88, 115107 (2013).

[33] U. Fano, Phys. Rev. 124, 1866 (1961).

[34] A. E. Miroshnichenko, S. Flach, and Y. S. Kivshar, Rev. Mod. Phys. 82, 2257 (2010).

[35] J. Sun, A. Ruzsinszky, and J. P. Perdew, Phys. Rev. Lett. 115, 036402 (2015). 